\title{
Motivating future directions of behavioral medicine
}

\author{
Claudio R. Nigg ${ }^{1}$ - Danielle E. Jake-Schoffman ${ }^{2}$ E. Amy Janke ${ }^{3}$
}

Received: December 2, 2016/Accepted: December 9, 2016/Published online: December 27, 2016

(C) Springer Science+Business Media New York 2016

Behavioral medicine stands to make a significant impact on the future horizons of health and healthcare. The top 10 causes of mortality worldwide are heart disease, stroke, chronic obstructive lung disease, lower respiratory infections, trachea bronchus and lung cancers, HIV/AIDS, diarrheal diseases, diabetes mellitus, road injuries, and hypertensive heart disease [World Health Organization (WHO), 2012]. In 2011, non-communicable diseases (NCDs) were responsible for $68 \%$ of all deaths globally and $7 / 10$ causes of mortality are related to health behaviors (WHO, 2012). Behavior matters to prevent chronic disease and to minimize morbidity and mortality when it occurs.

Behavioral medicine is a field of research and practice encompassing biological, behavioral, psychological, and social sciences relevant to health and illness (Fisher et al., 2011). It is a broad field and is not aligned with a specific profession, medical specialty, or discipline. Behavioral medicine is at the intersection between communication science, consumer education, industry, medicine, public health, and technology. It includes a variety of techniques applied to research, prevention, and treatment in the health area.

The Society of Behavioral Medicine (SBM) is a professional organization comprised of members with a broad

Claudio R. Nigg

cnigg@ hawaii.edu

1 Office of Public Health Studies, University of Hawaii, 1960 East-West Road, Honolulu, HI 96822, USA

2 Division of Preventive and Behavioral Medicine, University of Massachusetts Medical School, Worcester, MA, USA

3 Department of Behavioral and Social Sciences, University of the Sciences, Philadelphia, PA, USA range of expertise in this field, which is reflected in the Special Interest Groups (SIGs; SBM, 2016). These SIGs represent techniques, populations, behaviors, and outcomes of interest in behavioral medicine, and are (in alphabetical order): Aging, Behavioral Informatics and Technology, Cancer, Child and Family Health, Complementary and Integrative Medicine, Diabetes, Ethnic Minority and Multicultural Health, Evidence-Based Behavioral Medicine, Health Decision Making, Integrated Primary Care, Military and Veterans' Health, Multi-Morbidities, Multiple Health Behavior Change, Obesity and Eating Disorders, Optimization of Behavioral Interventions, Pain, Physical Activity, Population Health Sciences, Sleep, Spirituality and Health, Student, Theories and Techniques of Behavior Change Interventions, Violence and Trauma, and Women's Health (http://www.sbm.org/about/special-interest-groups). These SIGs are member-driven groups interested in the specific topics that are motivated to advance and impact the field.

The future of behavioral medicine lies in our ability to transform our science into action. We must lead the way in underscoring the dynamic nature of behavioral medicine and create synergy across disciplines to advance the nation's health and provide direction for the next generation of leaders in the field. Therefore, we assembled this landmark special issue as a call for action to promote research advancing the science to inform policy and practice. This is intended for the broader behavioral medicine community on the wide range of topics represented by a selection of the SBM SIGs. All SBM SIGs were invited to contribute and submission was voluntary. The participating SBM SIGs assembled a leadership team to discuss the most pertinent areas of future growth for 
their respective areas of study, then put forth a concept abstract. These abstracts received editorial review, upon which the SIGs developed a manuscript to describe their vision for the future of their field. Manuscripts were subjected to editorial and peer review and resulted in the representation of $15 / 24(62.5 \%)$ SIGs in this special issue. While the SIGs represent diverse areas of study, several common themes emerged as critical areas for future research across the articles.

\section{Behavioral medicine methodology: design, implementation, and dissemination}

Methodological issues represent a vital area of research in behavioral medicine where substantial progress can be made. The ongoing importance of rigorous methodology characterized by clearly operationalized concepts and measurement approaches must still be underscored to improve the validity and reliability of results across research areas (Geller et al., 2016; Park et al., 2016). Mechanisms of change should be explored to fully understand the process by which a lifestyle or behavior-such as religion and/or spirituality_impacts health outcomes (Park et al., 2016). Behavior change ontologies-a clearly defined, shared vocabulary of terms and the specific relationships between those terms-have the potential to transform behavioral science field to an integrative field where data are compared and contrasted (Larsen et al., 2016). Ontologies could lead to new approaches to hypothesis generation and knowledge discovery in our field.

Indeed, while the randomized clinical trial (RCT) has long been considered the pinnacle in understanding the effectiveness of treatment, there is an increasing shift away from these designs in favor of more innovative flexible approaches that focus on "real-world" implementation and patient-centered outcomes. Vogel et al. (2016) argue the RCT with patient-level randomization cannot appropriately assess the effectiveness of integrated behavioral health, and underscore the value in quasi-experimental, qualitative, and mixed methods designs to addressing gaps in our knowledge base. Buscemi et al. (2016) argue for a multiphase optimization strategy (MOST) approach to optimize multicomponent behavioral and bio-behavioral interventions. Inspired by engineering, MOST offers a framework that includes three phases to inform clinical trial design: preparation, optimization, and evaluation (Collins et al., 2005). While preparation and evaluation are common components of trial design, the focus on optimization or "the process of finding the best possible solution, subject to the given constraints" sets MOST apart from traditional
RCT designs. Most designs may be particularly applicable in the eHealth or technology based interventions (Moller et al. 2016).

Alcaraz et al. (2016) introduce the ConNECT Framework to link behavioral medicine and health equity in order to achieve equitable health and outcomes in the 21 st century. ConNECT's five broad and synergistic principles: (1) Integrating Context; (2) Fostering a Norm of Inclusion; (3) Ensuring Equitable Diffusion of Innovations; (4) Harnessing Communication Technology; and (5) Prioritizing Specialized Training are promising in promoting health equity across behavioral medicine areas.

The importance of dissemination to achieve maximal health impact was shared across several articles (e.g., Geller et al., 2016). While there is a large evidence base demonstrating that lifestyle interventions can aid in prevention of chronic disease, not enough people are benefiting from these programs (Stetson et al., 2016). What we need are low-cost, high-impact approaches to disseminating this knowledge on a larger scale. And, to achieve the goal of low-cost interventions, we need to better understand how cost is related to dissemination and intervention sustainability in different healthcare systems (Vogel et al., 2016). Buscemi et al. (2006) highlight the Reach, Effectiveness-Adoption, Implementation, Maintenance (REAIM) framework as a useful model to guide such dissemination. RE-AIM is designed to improve the quality, rapidity, and impact of translational research by guiding researchers in Reaching their intended population, focusing on Efficacy or effectiveness, increasing intervention Adoption, and ensuring Implementation consistency and intervention Maintenance over time (Glasgow et al., 1999). Lewis et al. (2016) further underscore the value of the REAIM framework, and provide several examples of how REAIM has been used within physical activity dissemination studies.

The burden of chronic disease is large, and many individuals are working to manage consequences of multiple chronic health conditions simultaneously, necessitating the need for broadly applicable lifestyle solutions. Geller et al. (2016) describe the emerging directions of the field of multiple health behavior change, including a need for a better understanding of the ways that cross-motivations could be employed to bolster the impact of multiple health behavior change interventions as well as a need to shift the focus of the field to sustainable interventions that can impact long-term change and maintenance of those changes. Vogel et al. (2016) describe the potential for religiousness and spirituality to aid in the prevention and treatment of various diseases, specifically describing their effects in addressing cardiovascular disease, cancer, and substance abuse. With a movement toward patient-centered care, informed and shared decision making is a critical part 
of healthcare delivery and quality. However, there has been little consensus about what constitutes a good medical decision, leading experts from SBM and the Society for Medical Decision Making to hold structured conference sessions to discuss this issue and how to better define it. Hamilton et al. (2016) describe the results of these sessions, including brainstorming about the concepts that influence decision making, as well as priority research areas to move the field forward towards a broad definition of a good medical decision.

\section{Intervention across the lifespan}

Another theme for the future behavioral medicine research identified by several articles is considering a lifespan, developmental approaches to health, and understanding how needs may differ across life stages. The importance of consideration of changes across the lifespan is highlighted in several papers with a focus on critical timepoints for intervention, identified in this special issue as (but not limited to) the interconception period, childhood, early adulthood, and transitions in military service and life as a veteran.

While the medical community recognizes the importance of transitions such as the interconception period for both maternal and infant health outcomes, there is a lack of interventions to specifically address maternal weight and stress management during this critical period. To this end, Huberty et al. (2016) review the current evidence for interventions during interconception and offer recommendations for future research including programs tailored for interconception, educational campaigns for healthcare providers about interconception, and studies designed to demonstrate the long-term public health benefits of intervening in interconception. Fuemmeler et al. (2016) describe the impact of childhood obesity and early trauma/ violence on the trajectory of children's health using a life course perspective. The life course perspective asks that researchers and health practitioners take a broad holistic social ecological approach to intervening for health promotion, and consider risk and protective factors across the domains of influence. Similarly, transitions associated with emerging adulthood present unique challenges, risks, and needs for intervention and prevention, particularly with regard to chronic diseases such as diabetes (Stetson et al., 2016). The health of the millions of military service members and veterans in the US is of great concern, especially given the fact that military service members tend to be healthier than the general population upon entry but have equivalent or worse health than civilians long term. Haibach et al. (2016) describe the ongoing health promotion efforts of the Department of Defense and Department of Veterans Affairs, with special attention to the need for health promotion research focused on key transition periods in both military service and veteran life.

\section{A focus on context: person in place matters}

There is a clear need for culturally, contextually, and linguistically/literacy relevant intervention approaches and content. Health disparities remain particularly salient in key areas, such as increasing physical activity, reducing risk for diabetes, and addressing obesity. Behavioral medicine research too often neglects to examine key areas related to culture and context-such as religion and spirituality-that have a significant impact on well-being (Park et al., 2016).

While there are many disease management programs that have demonstrated efficacy-such as diabetes prevention and management programs-little is known about how environmental and contextual factors influence disease burden and intervention success. Stetson et al. (2016) describe the need for ecological models of diabetes that include an understanding of policy and place, and research designs that have adequate minority representation. Buscemi et al. (2016) also underscore the importance of place and environment on health outcomes.

Integrative primary care is a model that is growing rapidly and being implemented widely. However, Vogel et al. (2016) remind us that our understanding of how minority populations can be best served by this model is still lacking. Furthermore, the most rigorous implementation studies of this model tend to occur in very specific environments (such as VA hospitals, or highly centralized health maintenance organizations) that do not represent the care provided to the majority of individuals in the U.S.

\section{Use of technology in behavioral medicine research}

Traditional intervention modalities that typically require face-to-face visits with professional staff in specific care settings have shown to be impractical for some populations. Kozak et al. (2016) review the current literature on eHealth weight management interventions, including their potential to reduce barriers to reach and engagement see with traditional interventions (Kozak et al., 2016). Challenges and methodological considerations for eHealth interventions are also discussed as important considerations for the future of this area of research.

One area where technology has a broad, consumer-based reach and potential for significant growth is in improving engagement in physical activity and reducing sedentary behavior. While technology has contributed to increases in 
sedentary behavior, Lewis et al. (2016) also outline how these tools can be used in innovative ways to reduce sedentary behavior and increase physical activity. Large randomized trials, a lifespan approach, and a commitment to theoretically-driven, evidence-based intervention components targeting long-term outcomes are key to achieving these two future aims.

Relatedly, technology has a very real potential to revolutionize theory refinement by leveraging real-time objective data and big data generated and stored digitally. Moller et al. (2016) present the opportunities provided by three of the cutting edge methods for leveraging the potential of digital platforms: optimization designs, dynamic systems modeling, and social network analysis.

Technology may be a particularly powerful tool to help patients with chronic disease management that requires multiple and complicated tasks, such as in diabetes management, but research is need to understand how to optimize these tools. Also, to appropriately use these tools we must understand their benefits alongside potential drawbacks, and we must explore the psychological and behavioral implications of these innovations on individuals who use them (Stetson et al., 2016). Technology may not be the right tool for everyone, and while it holds promise its effects may not be unilaterally positive.

\section{Behavioral medicine training}

To realize the future potential of behavioral medicine research, training of future behavioral medicine experts also needs to adapt. The Student SIG conducted a survey of SBM members addressing this very topic and highlight seven key areas that emerged as suggestions for improved preparation of the next generation of behavioral medicine scientists and practitioners. These suggestions include training in grant writing, interdisciplinary teamwork, advanced statistics and methods, how to craft an evolving research program, developing publishable products from coursework, understanding the evolution of and use of theory, and considering nontraditional career paths (Goldstein et al., 2016). Programs of training are well guided to consider these areas to increase the value of their content.

Training should be an important focus of empirical study. While provider training likely influences patient outcomes in important ways, this area of research remains largely unexamined. For example, Vogel et al. (2016) describe the variety of training represented in integrated primary care models and the lack of research to determine what are appropriate levels of and targets for education in these environments and what types of training and backgrounds produce the most optimal patient outcomes.
Indeed, healthcare is increasingly stressing the need for inter-professional practice and communication, behavioral medicine should be involved in these efforts as we have a unique skill set that can contribute to interprofessional effectiveness and measurement of team functioning (Vogel et al., 2016).

\section{A call to action}

As evidenced by the impressive collection of articles in this landmark issue we (the guest editors) are motivated and encourage researchers in our, and related and unrelated fields to collaborate and move the behavioral medicine field forward. There are numerous important areas highlighted which are vital to the well-being of all and represent research programs, and potential career areas. However, we should not be limited to just these topics highlighted in this special issue, but really use this call to forge new territories and collaborations about innovative areas such as punctuated equilibrium theory from physics; microgrants from economics; and design principles from holistic infrastructure architecture and planning just to name a few. We hope you enjoy reading these articles and that they inspire you to advance our science.

Acknowledgements The authors would like to acknowledge the inspiration of Marian Fitzgibbon for our efforts.

\section{References}

Alcaraz, K. I., Sly, J., Ashing, K., Fleisher, L., Gil-Rivas, V., Ford, S., et al. (2016). The ConNECT Framework: A model for advancing behavioral medicine science and practice to foster health equity. Journal of Behavioral Medicine. doi:10.1007/s10865-016-97804

Buscemi, J., Janke, E. A., Kugler, K. C., Duffecy, J., Mielenz, T. J., St. George, S. M., et al. (2016). Increasing the public health impact of evidence-based interventions in behavioral medicine: New approaches and future directions. Journal of Behavioral Medicine. doi:10.1007/s10865-016-9773-3

Collins, L. M., Murphy, S. A., Nair, V. N., \& Strecher, V. J. (2005). A strategy for optimizing and evaluating behavioral interventions. Annals of Behavioral Medicine, 30, 65-73. doi:10.1207/ s15324796abm3001_8

Fisher, E. B., Fitzgibbon, M. L., Glasgow, R. E., Haire-Joshu, D., Hayman, L. L., Kaplan, R. M., et al. (2011). Behavior matters. American Journal of Preventive Medicine, 40, e15-e30. doi:10. 1016/j.amepre.2010.12.031

Fuemmeler, B. F., Behrman, P., Taylor, M., Sokol, R., Rothman, E., Jacobson, L. T., et al. (2016). Child and family health in the era of prevention: New opportunities and challenges. Journal of Behavioral Medicine. doi:10.1007/s10865-016-9791-1

Geller, K., Lippke, S., \& Nigg, C. R. (2016). Future directions of multiple behavior change research. Journal of Behavioral Medicine. doi:10.1007/s10865-016-9809-8

Glasgow, R. E., Vogt, T. M., \& Boles, S. M. (1999). Evaluating the public health impact of health promotion interventions: The RE- 
AIM framework. American Journal of Public Health, 89, $1322-1327$.

Goldstein, C. M., Minges, K. E., Schoffman, D. E., \& Cases, M. G. (2016). Preparing tomorrow's behavioral medicine scientists and practitioners: A survey of future directions for education and training. Journal of Behavioral Medicine. doi:10.1007/s10865016-9758-2

Haibach, J. P., Haibach, M. A., Hall, K. S., Masheb, R. M., Little, M. A., Shepardson, R. L., et al. (2016). Military and veteran health behavior research and practice: Challenges and opportunities. Journal of Behavioral Medicine. doi:10.1007/s10865-016-9794$\mathrm{y}$

Hamilton, J. G., Lillie, S. E., Alden, D. L., Scherer, L., Oser, M., Rini, C., et al. (2016). What is a good medical decision? A research agenda guided by perspectives from multiple stakeholders. Journal of Behavioral Medicine. doi:10.1007/s10865-0169785-z

Huberty, J., Leiferman, J. A., Kruper, A. R., Jacobson, L. T., Waring, M. E., Matthews, J. L., et al. (2016). Exploring the need for interventions to manage weight and stress during interconception. Journal of Behavioral Medicine. doi:10.1007/s10865-0169813-z

Kozak, A. T., Buscemi, J., Hawkins, M. A. W., Wang, M. L., Breland, J. Y., Ross, K. M., et al. (2016). Technology-based interventions for weight management: Current randomized controlled trial evidence and future directions. Journal of Behavioral Medicine. doi:10.1007/s10865-016-9805-z

Larsen, K. R., Michie, S., Hekler, E. B., Gibson, B., Spruijt-Metz, D., Ahern, D., et al. (2016). Behavior change interventions: The potential of ontologies for advancing science and practice. Journal of Behavioral Medicine. doi:10.1007/s10865-016-9768-0
Lewis, B. A., Napolitano, M. A., Buman, M. P., Williams, D. M., \& Nigg, C. R. (2016). Future directions in physical activity intervention research: Expanding our focus to sedentary behaviors, technology, and dissemination. Journal of Behavioral Medicine. doi:10.1007/s10865-016-9797-8

Moller, A. C., Merchant, G., Conroy, D. E., West, R., Heckler, E., Kugler, K. C., \& Michie, S. (2016). Applying and advancing behavior change theories and techniques in the context of a digital health revolution: Proposals for more effectively realizing untapped potential. Journal of Behavioral Medicine. doi:10. 1007/s10865-016-9818-7.

Park, C. L., Masters, K. S., Salsman, J. M., Wachholtz, A., Clements, A. D., Salmoirago-Blotcher, E., et al. (2016). Advancing our understanding of religion and spirituality in the context of behavioral medicine. Journal of Behavioral Medicine. doi:10. 1007/s10865-016-9755-5

Society of Behavioral Medicine. (2016). About. Retrieved from http:// www.sbm.org/about. Accessed 11/18/2016.

Stetson, B., Minges, K. E., \& Richardson, C. R. (2016). New directions for diabetes prevention and management in behavioral medicine. Journal of Behavioral Medicine. doi:10.1007/s10865016-9802-2

Vogel, M. E., Kanzler, K. E., Aikens, J. E., \& Goodie, J. L. (2016). Integration of behavioral health and primary care: Current knowledge and future directions. Journal of Behavioral Medicine. doi:10.1007/s10865-016-9798-7

WHO. (2012). The top 10 causes of death. http://www.who.int/ mediacentre/factsheets/fs310/en/. Accessed 11/13/2016. 